\title{
Neutrosophic -Based Bayesian Neural Network for Handling Data Overlap Problem In Classification
}

\author{
Okpako, A.E \\ Department of Mathematical Sciences \\ Edwin Clark University \\ Kiagbodo, Delta State, Nigeria. \\ E-mail: okpako.ejaita@gmail.com \\ Ako, R.E. \\ Department of Mathematical Sciences \\ Edwin Clark University \\ Kiagbodo, Delta State, Nigeria. \\ E-mail: ochukorita2@gmail.com
}

\begin{abstract}
Overlapping region is a region in data where at least two different classes $\mathrm{Ca}$ and $\mathrm{Cb}$ simultaneously exhibit probability densities greater than zero. Overlapping problem has been a serious problem in classification system shown to have negative effect on the performance of even re-known classifiers by several researchers.Neural network is very poor in representing uncertainty as well as having uninterpretable black-boxes, lacking in transparency, difficult to trust. It is therefore pertinent to develop a system to work making use of neural network to effectively handle cases of non-linear decision boundaries poised with overlap problem amidst the varied causes of overlapping problems in datasets as well as augment it with transparency and trust metric so as ensure that it is interpretable to an extent. Object Oriented Analysis and Design Methodology was used while the implementation was done using WEKA and Java on a Netbeans platform. Experimental results show that Neutrosophic-Based Bayesian Neural Network (|NBBNNS) with an accuracy of $96.41 \%$ using a confusability measurement threshold of 0.003278 performed better than the conventional Neural Network had an accuracy of $72.45 \%$ using ILPD dataset; Accuracy of $100 \%$ was observed for both Wine and Iris datasets respectively. Our system was also used to differentiate between ILDP positive instances and Hepatitis Positive instances shown to have concept overlapping problem with accuracy of $97.30 \%$. This clearly shows that Neutrosophic-Based Bayesian Neural Network (BNNS) is a promising classifier or system for classification of datasets prone to overlap problems.
\end{abstract}

Keywords: Neutrosophic logic, Bayesian Neural Network, Confusability measurement, fuzzy logic

CISDI Journal Reference Format

Okpako, A.E. \& Ako, R.E. (2019): Neutrosophic -Based Bayesian Neural Network for Handling Data Overlap Problem in Classification. Computing, Information Systems, Development Informatics \& Allied Research Journal. Vol 10 No 1.Pp 29-50.

Available online at www.cisdijournal.org

DOI Affix - https://doi.org/ 10.22624/AIMS/CISDI/V10N1P4 


\section{INTRODUCTION}

Classification has been extensively used in statistical literature and machine learning as well as in data mining and in each of these cases they are either referred to as discrimination or supervised learning. Classification is traditionally seen as a process of building a function or a rule from a training data and uses these rules to designate new data into their respective groups. Several classification algorithms have been developed and have been successfully applied to many domains but as good as they are, researchers are still seeking ways to maximizing or optimizing their classification performance in relation to practical implementations. Class imbalance when considered in a two class dataset, is a situation where one of the classes is heavily under-represented in comparison to the other class otherwise known as the majority one.

The concept of class imbalance has attracted a lot of researchers' attention because of the cost associated to misclassification of examples from the minority when considered in a real life environment. Maximizing the accuracy is not an easy one in real world data where one class might be represented by a large number of examples while the other is underrepresented owing to the fact that they have fewer examples such as Hepatitis Positive patient in Niger Delta, oil spill detection, fraud detection, network intrusion to mention a few. Most classification algorithm or techniques try to minimize the total misclassification error rate which is mostly attributed to cases of overlapping between the two classes. They ignore the differences between types of misclassification error in particular and they assume that all misclassification error cost equally.

The remainder of this paper is organized as follows: Section 2 gives a literature review of the overlap problem in data classification. Section 3 describes the theoretical concepts underlying the proposed system. Section 4 presents the experiments and evaluation. Conclusion and future work are given in the final section.

\section{LITERATURE REVIEW}

Class overlap has been touted by researchers in machine learning to have negative impact on the classification performance of various classifiers with varying magnitude. Class overlap region $S \in R_{n}$ in an $n$-dimensional feature space $R_{n}$ can be described as a region where at least two different classes $C_{a}$ and $C_{b}$ simultaneously exhibit probability densities greater than zero, i.e., $P\left(x \mid C_{a}\right)>0$ and $P\left(x \mid C_{b}\right)>0, \forall x \in$ Sand $a=b$ as opined by Kretzschmar et al (2003). There has been a natural quest by researchers to uncover the hidden patterns that may be overlapped with data from two different classes. According to Haitao et al (2013), the overlap problem is handled using two perspectives known as sample overlapping and concept overlapping perspective.

i. Sample overlapping- This is a micro view point where some sample from one class have similar characteristics or overlap with samples from the other class in feature space.

ii. Concept overlapping- This is a macro viewpoint where samples from one class and the other class have overlapping region in the feature space.

Several research works have pointed out that performance degradation is not solely dependent on class imbalance; rather it depends on other complexities. Japkowicz (2000) opine that in case of feed forward neural networks, class imbalance does hinder its performance especially when the data complexity increases. As a continuation of that, Japkowicz and Stephen (2002) discovered that as the level of data complexity increases, the class imbalance factor begins to affect the generalization capability of a classifier. Japkowicz (2001) also identified the distribution of the data within each class. Other factors are also identified such as small disjuncts by Weiss and Provost (2003), data duplication as identified in the work of Kolez et al (2003), and the reason for this research work is the identification of density and overlap complexity as a factor by Visa and Ralescu (2003). 
Prati et al (2004), opined that although there is no direct relationship or correlation between performance degradation and class imbalance rather they pointed out that the degradation of performance of classifiers is related to other factors. In line with the work of Prati et al (2004), Okamoto and Yugami (2003) opined that size of data set is one of such factors.

Mazurowski et al (2008) investigated the effect of class imbalance in training data when developing neural network classifiers for computer aided medical diagnosis and showed that classifier performance deteriorates in a class imbalance environment or scenario. They stressed that uncertainty and imprecision are the striking problem in developing expert systems in medicine. Garcia et al (2008) analyzed the influence of overlapping for the learning ability of kNN classifiers in imbalanced classification and opined that the main problem is the separability between the classes rather than just the skewed distribution. Denil and Trappenberg (2010) examined the effects of overlap and imbalance on the complexity of the learned model and demonstrated that overlapping is a far more serious factor than imbalance in this respect. They opined that these two problems acting in concert cause difficulties that are more severe than one would expect by examining their effects in isolation. In order to do so, they used synthetic datasets for classifying with a SVM where they vary the imbalance ratio, the overlap between classes and the imbalance ratio and overlap jointly. Their results showed that when the training set size is small, high levels of imbalance cause a dramatic drop in classifier performance, explained by the presence of small disjuncts.

Overlapping classes causes a consistent drop in performance regardless of the size of the training set. However, with overlapping and imbalance combined, the classifier performance is degraded significantly beyond what the model predicts. Luengo et al (2011) empirically extracted some interesting findings on real world datasets. Specifically, they depicted the performance of the different datasets ordered according to different data complexity measures (including the Imbalance Ratio) in order to search for some regions of interesting good or bad behaviour. They could not characterize any interesting behaviour related to Imbalance Ratio, but they did for other metrics that measure the overlap between the classes.

\subsection{Theoretical Framework:}

Central to the proposed algorithm is the machine learning algorithm called neural networks which is used for the classification task after the suitable number of features have been selected through the feature selection component; though in this system, feature selection was not used. The distributions of each of the instances are actually calculated in order to determine the reliability metric to measure the indeterminacy in the datasets. The varied pattern and values are analyzed and a suitable threshold is used to adequately classify the instances into their respective classes.

\subsubsection{Neural Network and Bayesian Neural network:}

This is a black box supervised learning algorithms used widely in designing and programming several intelligent systems whose working principle is in tandem with natural biological neurons. Neural network are based on simulated neurons which are joined together in a variety of ways to form networks. Neural network resembles the human brain in the following two ways: -

a) A neural network acquires knowledge through learning

b) Neural networks knowledge is stored within the interconnection strengths known as synaptic weight.

Networks are typically organized in layers. Layers are made up of a number of interconnected nodes which contain an activation function. Patterns are presented to the network via the input layer, which communicates to one or more hidden layers where the actual processing is done via a system of weighted connections. It should be noted that neural network is very poor in representing uncertainty as well as having uninterpretable black-boxes, lacking in transparency and difficult to trust. 
Instead of considering a single answer to a question, Bayesian methods on the other hand allow us to consider an entire distribution of answers. With this approach we can naturally address issues like: - regularization (over fitting or not), ' model selection / comparison, without the need for a separate cross-validation data set. With these techniques we can also put error bars on the output of the network, by considering the shape of the output distribution $\mathrm{p}(\mathrm{y} \mid \mathrm{D})$.

\subsection{Neutrosophic Logic and Fuzzy Logic}

It is an open truth that scientists in the quest to unravel the underlying course and effect of any phenomenon have always look for typical and normal phenomenon but Medical sciences is however orthogonal in this regard as it looks into atypical, abnormal and morbid phenomenon. It is the central purpose of medical science to finding a law or relation for these abnormal, atypical and sometimes morbid phenomena in order to characterize such situations. Ludwik Fleck (1896-1961), a polish philosopher and physician was the first to notice this and rightly presented it in his book titled "Genesis and Development of a scientific fact" in 1926. He opined that there are some specific features of the medical way of thinking. He also went further to state that there was no sharp border between these phenomena. Smooth transitions in the space of diseases feed from one phenomenon and very small variations could be the reasons that the physician diagnoses a patient's with $\mathrm{x}$ instead of $\mathrm{y}$.

It is an established conception that physician tends to rely on experience and intuition and not on rational and strong rules to deduce from the patients' data to a disease. Fuzzy logic proposed by Lofti Zadeh on the other hand deals with the notion of degree of belongingness in which value ranges between 0 and 1 or true or false respectively. The degree is expressed as the membership function of a mapping from a set $A$ to the interval $[0,1]$ using a linguistic variable. Membership function denoted by $A_{s}(x)$ represents a unique fuzzy set $S$. This logic is quite different from previous logic theory because rather than considering the categorical boundaries of 1 and 0 only for classes, it seeks for the degree of membership into the respective classes or sets. If the value is 0 , it means that the element does not belong to the fuzzy set $\mathrm{S}$, otherwise 1 , which means that it belongs fully to the fuzzy set $\mathrm{S}$.

$$
\left.A_{s}(x): X \longrightarrow 0,1\right]
$$

Zadeh (1999) stated that "In a given pathology, denoted by S a set of symptoms, D a set of diagnoses and P a set of patients. What we call medical knowledge is a fuzzy relation, generally denoted by $R$, from $S$ to $D$ expressing associations between symptoms or syndromes, and diagnoses or group of diagnoses". The Neutrosophic Logic proposed by Smarandache (1995) is an extension of fuzzy logic which ensures that in addition to the membership to truth and falsity as presented in Zadeh's fuzzy logic, it caters for issues of uncertainty, ambiguity, redundancy, imprecision, undefinedness, unknown, incompleteness and other varied meanings of indeterminacy. It is a logic in which each proposition is estimated to have the percentage of truth in a subset $T$, the percentage of indeterminacy in a subset $I$, and the percentage of falsity in a subset $F$, where $T, I, F$ are defined above, is called Neutrosophic Logic. A Neutrosophic set $A$ in $X$ is characterized by a truth membership function TA, an indeterminacy- membership function $\mathrm{IA}$ and a falsity-membership function $F A$. TA(x), IA(x) and $F A(x)$ are real standard or non-standard subsets of $-0,1+[$.

\subsection{Class Conditional Density Functions}

The probability distribution is defined as a function over general sets of values, or it may refer to the cumulative distribution function. Probability density functions have been used in statistics and probability theory for the density of continuous variables whose value at any given time in the set of possible values taken at random can provide a relative likelihood that the value of the random variables would equal that sample. To put it in a more simple form, while the absolute likelihood from a continuous variable to take any particular value is 0 , the value of the PDF at two different samples can be used to infer, in any particular situation or event of a random draw of the variable, how much likely it is that the random variable would equal one sample compared to the other sample. 
This PDF is used to specify the probability of any sample falling into any class of the dataset as opposed to only one class. The PDF over the entire dataset or sample space is equal to 1.

\section{METHODOLOGY}

The use of effective and appropriate methods in facilitating projects enhances effectiveness and efficiency. The method applied in this study is the use of Object Oriented System Analysis and Design method (OOAD) where an existing system is studied from the perspective of objects and similar objects are grouped as classes; their properties are handled as fields while their behaviors are treated as the actions or methods within the same bundle of object. The choice of this methodology is clear since it is a method developed in Software Engineering during the last decades to develop computational models of reality and it is a type of tool needed when one deal with the development of complex computational applications.

\subsection{Data Collection}

Collection of quality data is an indispensable aspect of machine learning. This research work made use of data from online database(www.uci.com) which are available publicly which are collected from good research environment and have undergone proper organization ethics approval processes and available freely for research purposes. There is an inherent advantage in the use of this publicly available database which is the ability to make comparison between our methods and the existing methods by using the same dataset.

\section{Dataset}

We had earlier seen from literature that overlap of data has negative impact on the classification performance of data making the performance of good classifier to go south. Therefore we tried our model on well-known overlap poised datasets which are based on sample overlapping such as Iris, Wine and ILDP so as to validate our model against the datasets which were used in existing systems. The datasets used in this work are presented in Table 1 below.

In view of evaluating and validating our proposed model for classifying overlap datasets based on concept overlapping, two disease datasets from $\mathrm{UCl}$ standard datasets whose attributes show considerable overlap and confusing in terms of diagnosis to a novice physician were used. The first dataset is Liver disorder dataset (ILPD) which contains 583 instances of 11 attributes/symptoms classified as either patient or non-patient while the second one is Hepatitis which contains 155 instances of 20 attributes classified as either DIE or LIVE. These datasets are downloaded from the University of California at Irvine website (www.uci.com).

Table 1: Datasets Used in this Study

\begin{tabular}{|c|c|c|c|c|}
\hline Name & No. of Classes & No. of Features & Feature Type & Size of Samples \\
\hline Diabetes & 2 & 8 & Numeric & 768 \\
\hline Hepatitis & 2 & 19 & Numeric & 155 \\
\hline Iris & 3 & 4 & Numeric & 150 \\
\hline Liver Disorder & 2 & 10 & Numeric & 583 \\
\hline Wine & 3 & 13 & Numeric & 178 \\
\hline
\end{tabular}




\section{2: Architecture of the Proposed System}

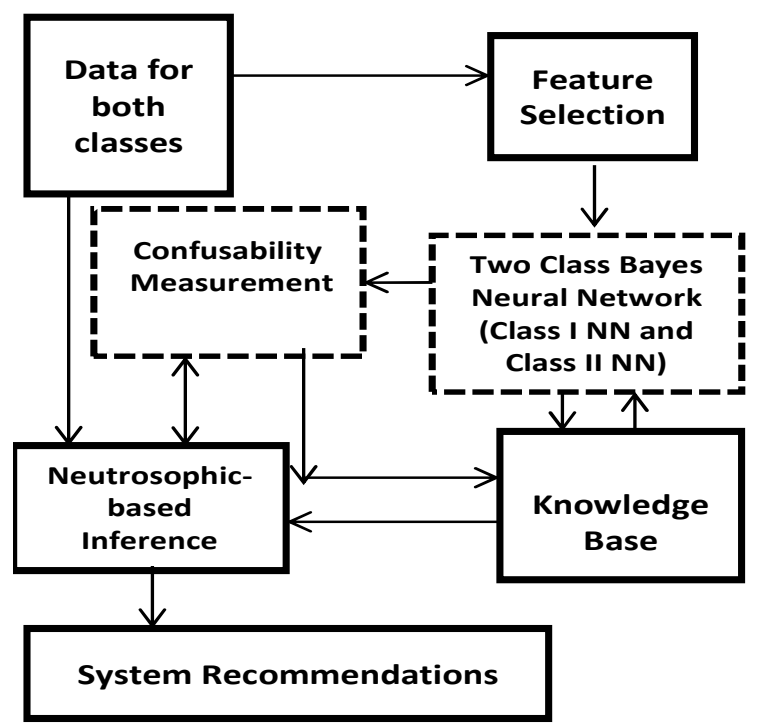

Fig 1: Proposed system

\subsection{Algorithm of the Proposed System}

The algorithm for the proposed is composed of four phases, which are Phase I (Feature Selection-optional) shown in Figure 2, Phase II( Network training phase) is shown in Figure 3 while Phase III( Confusability Measurement) and Phase IV (Testing Phase) is shown in Figure 4 respectively.

\section{Feature Selection (optional):}

Step 1: Generation of initial features, fitness at $i=$ fitnes (i) where $i=0$

Step 2: Perform cross over operation on the features

Step 3: Perform mutation on them

Step 4: feed the features into the two neural networks

Step 5: Calculate the fitness value by Error=Target-output;

\section{Fitness $\mathrm{i}=1 / \mathrm{error}$}

Step 6: Evaluate fitness in order to select features-

a. If fitness( $i)<$ fitness( $i+1)$, take features( $i+1)$ else take features(i)

b. Go to step 2, and increment $\mathbf{i}$ to $\mathbf{i}+\mathbf{1}$ until maximum fitness is reached

Fig. 2: Phase I-Pseudo code for feature selection 
Neural Network Training Phase:

Step 7: The values of selected features in Step 6 should be fed into neural network (Class I and Class II NN)

Step 8: Calculate the Error=Target-Output for both the Class I and Class II which is denoted by $\mathbf{e}^{\mathbf{t}}$ and $\mathbf{e}^{\mathbf{f}}$ for all feature vectors -feature $(\mathrm{N})$ for every instance

Step 9: Calculate the local gradient for nodes in each netw

Step 10: Calculate the hidden error of the network;

Step 11: Adjust the weights of the network using the learning rule until Learning is complete.

Fig. 3: Phase II-Pseudo Code for neural network training phase

\section{Confusability Measurement:}

Step 12: Get the degree of belief in Class I denoted as $\operatorname{Pr}$ (Class $1 / \mathrm{X}$ )

Step 13: Get the degree of belief in Class II denoted as Pr(Class II/X)

Step 14: Calculate Confusability Measurement $=1-\mid \operatorname{Pr}($ Class $1 / X)-\operatorname{Pr}($ Class $I / / X) \mid$

Step 15: Determine the threshold CM from the validation data.

\section{Testing phase:}

Step 16: Test the system putting CM and the complexity measurement from the test data into consideration.

Fig. 4: Phase III and IV- Pseudo code for confusability measurement and testing phase 


\section{EXPERIMENT AND RESULT ANALYSIS}

The Neutrosophic logic enable our proposed system to have a reliability metric which would act as a diagnostic criterion for data samples or points to be definitely included or definitely excluded in non-linear decision boundary cases. This metric quantifies any neutralities or inherent form of uncertainties or indeterminacy that can have negative impact in such classification. Thus the quantified indeterminacy is utilized to know the extent of quality in any predicted instance and ultimately improve the classification performance.

\subsection{Neural Network Implementation Properties}

The neural network properties used in this research are presented as follows:

* Input Neurons: The number of neuron is determined by the number of attributes for any datasets being considered. For example for Iris dataset we have four input neurons. The confusable disease diagnosis of ILPD and Hepatitis is made of 23 neurons being the set of features of both diseases.

* Hidden Neurons: The number of chosen hidden neurons is 3

* Learning Rate: We chose a learning rate of 0.1 for the datasets except Hepatitis dataset which is 0.3 after careful experimental analysis

* Momentum: We chose a momentum of 0.2 after careful experimental analysis

* Epoch: An epoch of 2000 was chosen as it gives a low training error for Iris and Wine dataset, while an epoch of 1750 was used for Hepatitis, Liver Disorder (ILPD) and Diabetes. The elbow method is used.

In the training procedure, the methods mentioned in algorithm is used to train the neural network, calculates the parameter of the sigmoid functions, correction models and estimate the threshold values of confusability measurement from validation instances which is consequently used for the testing instances.

In the testing procedure, for each instance, the confusability measurement is obtained from which a suitable threshold for confusability measurement is determined and used for assigning the class labels accordingly.

\subsubsection{Machine Learning Software Implementation Tools}

The java technologies are fast becoming one of the suitable machine learning enabled programming languages due to its vast arrays of machine learning supporting libraries that can be used with it such as weka.jar and encog.jar, just to mention a few.

The implementation tools used are:

1. Netbeans 7.3 Integrated Development Environment (IDE)

2. WEKA.jar (Waikato Environment for Knowledge Analysis version 3.8.1)

3. jcommons.jar,jmathplot.jar,jama.jar,jfreechart.jar

With the aid of these libraries, machine learning capability is infused into the java platform. A training set of 416 instances obtained from the UCI dataset of Indian Liver Patient Dataset was used and the system mean square error was recorded. A test data of 167 which represents $30 \%$ of the dataset is used to test and study the performance of the system by presenting the test pattern. 


\subsection{Experimental Results and Analysis}

There are two basic ways or approaches in class overlaps which also result in class confusability as opined by Haitao et al (2013) which are sample (Internal) and external (Concept). The internal exists within a datasets while the external exists between two different diseases or datasets. Our existing systems considered internal confusability in datasets making use of some re-known datasets poised with high overlaps.

Our proposed system was evaluated and/or validated using datasets that show high confusability internally. Some of such datasets that are known to be highly overlapping and were also used in our existing systems are Iris, Wine, Diabetes, and Indian Liver Patient datasets taken from our existing system in order to compare their performance. The result of our proposed system using the various datasets considered is shown in Table 2 while the bar chart of the result in shown in Figure 5.

Table 2: Result of Our Proposed System (NBDSS) on Various Datasets Considered

\begin{tabular}{|l|l|l|l|l|}
\hline Dataset & Author & Methods & F-measure & Accuracy \\
\hline Iris & Our work & $\begin{array}{l}\text { NBDSS } \\
\text { (Our Model) }\end{array}$ & 1.0000 & $100 \%$ \\
\hline Wine & Our work & $\begin{array}{l}\text { NBDSS } \\
\text { (Our Model) }\end{array}$ & 1.0000 & $100 \%$ \\
\hline Diabetes & Our work & $\begin{array}{l}\text { NBDSS } \\
\text { (Our Model) }\end{array}$ & 0.8100 & NA \\
\hline ILPD & Our work & $\begin{array}{l}\text { NBDSS } \\
\text { (Our Model) }\end{array}$ & 0.9462 & $96.41 \%$ \\
\hline
\end{tabular}




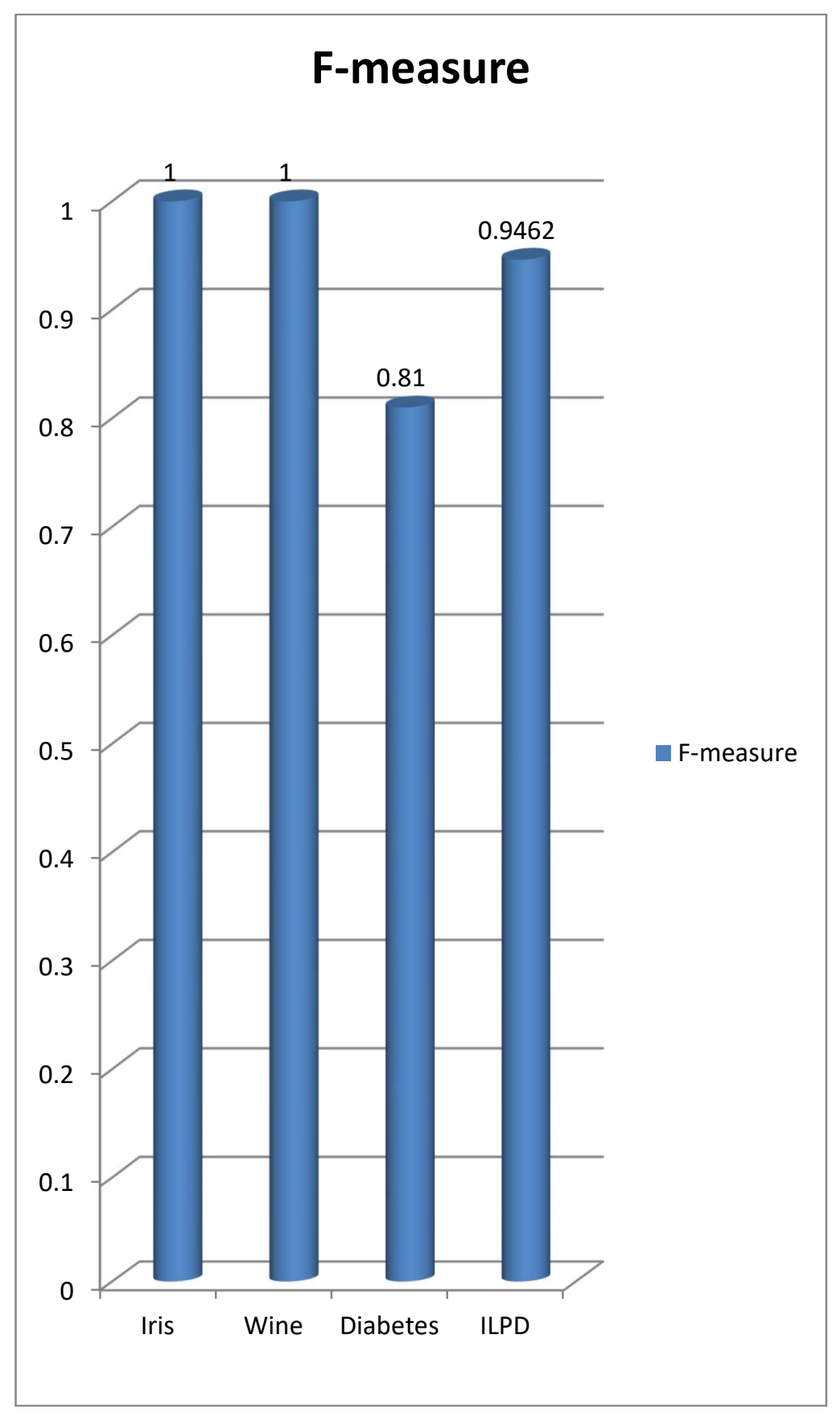

Fig 5: Performance analysis of proposed system using the various datasets 


\subsubsection{Performance Analysis of the Result of Our Proposed System with Existing Systems}

The summary of the dataset alongside their performance results is clearly shown in Table 3 in order to assay the performance of our proposed system while the bar chart of the table is shown in Figure 6.

Table 3: Summary of our Results in Relation to the Overlap Datasets of Existing Systems

\begin{tabular}{|c|c|c|c|c|}
\hline Dataset & Author(s)/Date & Methods & F-Measure & Resampling \\
\hline \multirow[t]{6}{*}{ Iris } & Sharma et al(2016) & K-NN & 0.9609 & \\
\hline & Sharma et al(2016) & C4.5 & 0.8900 & \\
\hline & Sharma et al(2016) & Naïve Bayes & 0.9400 & \\
\hline & Sharma et al(2016) & SVM & 0.9504 & \\
\hline & Sharma et al(2016) & Neural Network & 0.9523 & \\
\hline & Our work & NBDSS & 1.0000 & \\
\hline & & & & \\
\hline \multirow{6}{*}{ Wine } & Haitao et al(2013) & K-NN & 0.9790 & \\
\hline & Haitao et al(2013) & C4.5 & 0.9320 & \\
\hline & Haitao et al(2013) & Naïve Bayes & 0.9980 & \\
\hline & Haitao et al(2013) & SVM & 0.5790 & \\
\hline & Haitao et al(2013) & CANB & 0.9790 & \\
\hline & Our work & NBDS & 1.0000 & \\
\hline & & & & \\
\hline \multirow{11}{*}{ Diabetes } & Sharma et al(2016) & K-NN & 0.6330 & No \\
\hline & Sharma et al(2016) & C4.5 & 0.5455 & No \\
\hline & Sharma et al(2016) & Naïve Bayes & 0.2000 & No \\
\hline & Sharma et al(2016) & SVM & 0.6222 & No \\
\hline & Sharma et al(2016) & Neural Network & 0.6349 & No \\
\hline & Haitao et al(2013) & Naïve Bayes & 0.9980 & Yes \\
\hline & Haitao et al(2013) & CANB & 0.9790 & Yes \\
\hline & Haitao et al(2013) & SVM & 0.5790 & Yes \\
\hline & Haitao et al(2013) & C4.5 & 0.9320 & Yes \\
\hline & Haitao et al(2013) & K-NN & 0.9790 & Yes \\
\hline & Our work & NBDSS & 0.8100 & No \\
\hline \multirow{2}{*}{ ILPD } & Our work & MLP & 0.5925 & No \\
\hline & Our work & NBDSS & 0.9462 & No \\
\hline
\end{tabular}




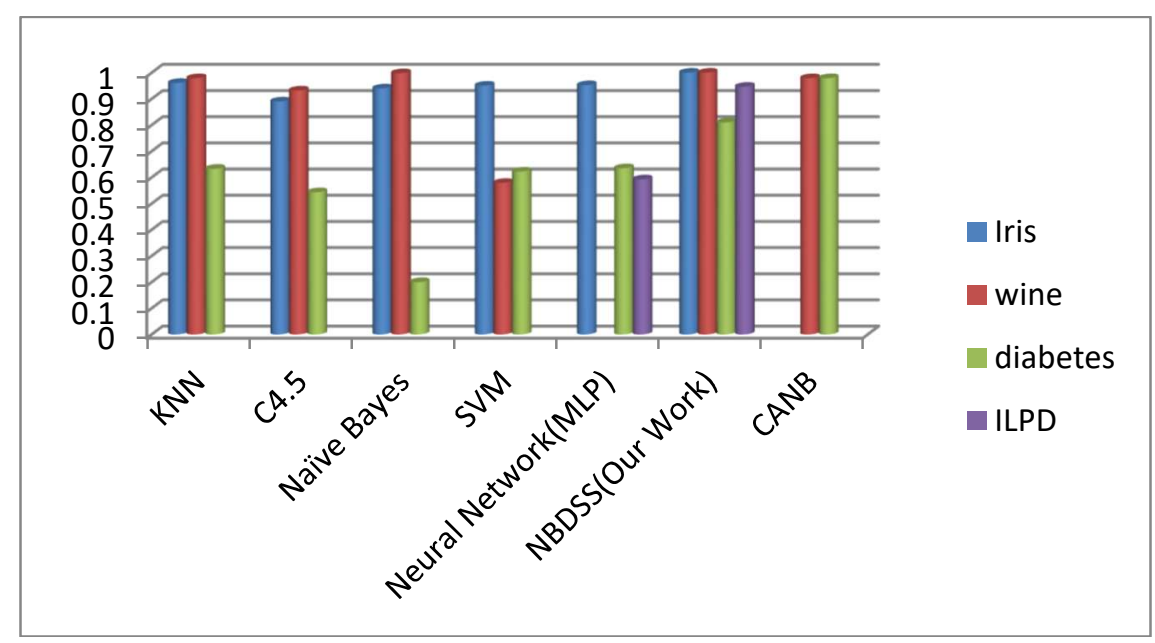

Fig. 6: Performance Analysis of the Result of Our Proposed System with Existing Systems

\subsubsection{Result of Our Proposed System on External Overlapping (ILPD /Hepatitis Datasets)}

It has been adequately pointed out that there is external confusability between Liver Disorder (ILPD) and Hepatitis. Our proposed system was also tested using this confusable disease to demonstrate the effectiveness of the proposed system in effectively diagnosing confusable disease. The performance result using the traditional Multilayer perceptron neural network is shown alongside our proposed system in Table 4 while Table 5 shows the comparative performance of the proposed system with the existing system of Mohammed et al (2015).

Table 4.8: Performance Comparison using the Proposed Model and Traditional Approach (MLP) for Diagnosing Confusable Disease (Indian Liver Patient Dataset/Hepatitis)

\begin{tabular}{|l|c|c|c|c|}
\hline Properties & \multicolumn{2}{|c|}{ Traditional Approach(MLP) } & \multicolumn{2}{c|}{ Our Proposed Model } \\
\hline & Class 1 & Class 2 & Class 1 & Class 2 \\
\hline Correctly classified & 41 & 32 & 39 & 36 \\
\hline $\begin{array}{l}\text { Incorrectly } \\
\text { classified }\end{array}$ & 0 & 5 & 2 & 1 \\
\hline TP rate & 1.0000 & 0.8650 & 0.9487 & 0.9722 \\
\hline FP Rate & 0.1350 & 0.0000 & 0.0278 & 0.0513 \\
\hline Precision & 0.8910 & 1.0000 & 0.9750 & 0.9474 \\
\hline Recall & 1.0000 & 0.8650 & 0.9487 & 0.9722 \\
\hline F-Measure & 0.9430 & 0.9280 & 0.9618 & 0.9596 \\
\hline Accuracy & \multicolumn{2}{|c|}{$93.5897 \%$} & & $96.15 \%$ \\
\hline $\begin{array}{l}\text { Weighted F- } \\
\text { measure }\end{array}$ & \multicolumn{2}{|c|}{0.935} & & 0.9607 \\
\hline
\end{tabular}


Table 5: Result using the Proposed Model for Diagnosing Confusable Diseases and Existing Technique of Mohammed et al (2015)

\begin{tabular}{|c|c|c|c|c|c|}
\hline Dataset & Authors & Methods & Accuracy & $\begin{array}{c}\text { No. } \\
\text { of } \\
\text { Attributes }\end{array}$ & $\begin{array}{c}\text { Overlap } \\
\text { Attributes }\end{array}$ \\
\hline $\begin{array}{c}\text { BUPA } \\
+ \\
\text { Hepatitis }\end{array}$ & $\begin{array}{c}\text { Mohammed et al } \\
(2015)\end{array}$ & $\begin{array}{c}\text { K-means } \\
\text { clustering } \\
+ \\
\text { SVM }\end{array}$ & 0.9943 & 25 & 2 \\
\hline $\begin{array}{c}\text { BUPA } \\
+ \\
\text { Hepatitis } \\
\text { ILPD } \\
+ \\
\text { Hepatitis }\end{array}$ & Our work & NBDSS & 1.0000 & 25 & 2 \\
\hline $\begin{array}{c}\text { (ILPD) } \\
+\end{array}$ & Our work) & Teural Network & 0.9360 & 22 & 6 \\
\hline \begin{tabular}{c} 
Hepatitis \\
\hline
\end{tabular} & NBDSS & $\mathbf{0 . 9 7 0 4}$ & $\mathbf{2 2}$ & 6 \\
\hline
\end{tabular}

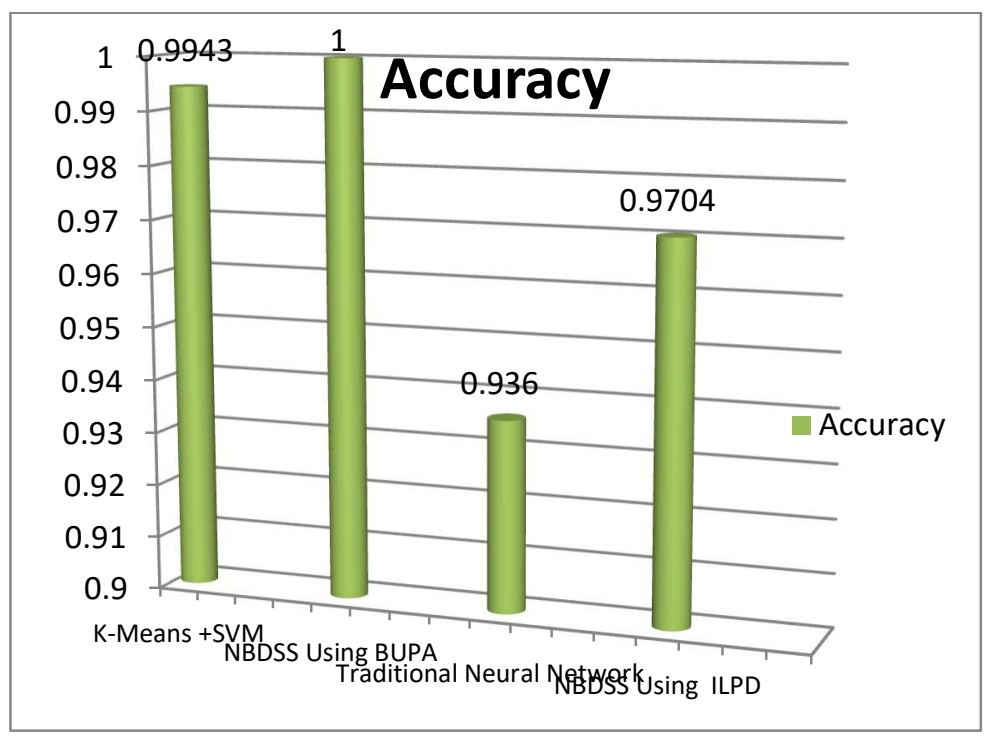

Fig 7: Performance comparison of confusable disease diagnosis 


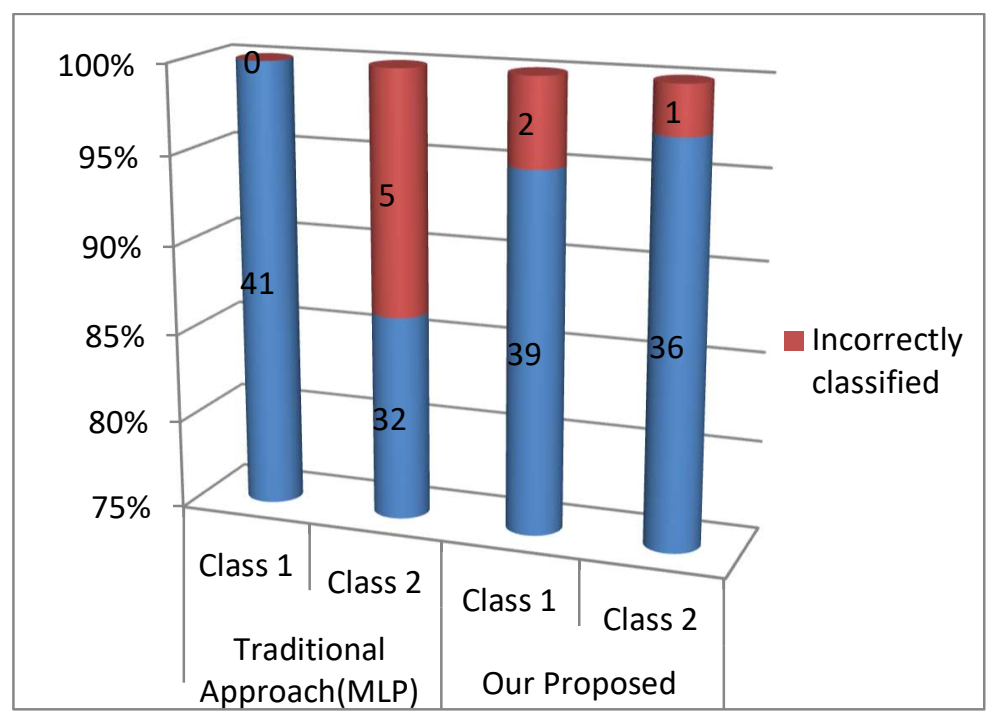

Fig. 8: Graph showing the comparative performance of the proposed model to the traditional model in terms of correctly and incorrectly classified instances

\subsection{Discussion of Results}

This section discusses the results of the various experiments done using our proposed model in relation to the various datasets.

\subsubsection{Internal Confusability}

Internal confusability is as a result of internal overlapping within classes of the same dataset. The datasets shown from research of existing systems to be overlapped and confusing are Iris, Wine, Diabetes, and Indian Liver Patient Dataset whose results are also given in the result section.

\section{(a) Discussion on Iris}

The iris dataset has three classes where one of them, Iris-Setosa, is linearly separable from the other two while IrisVirginica and Iris-Versicolor show considerable amount of overlap. Our model was tested using the dataset of IrisVirginica and Iris-Versicolor. The predictions of the dataset as well as the confusability measurement for all the testing instances are shown in Table 6. It is observed from the table that the confusability measurement of Iris-Virginica is higher than Iris-Versicolor and as such using a threshold of 0.0089 to separate the two classes.

Using the confusability measurement threshold of 0.0089 , our proposed model recorded a superlative performance of ROC (AUC) of 1(unity) as well as a high specificity and sensitivity with an F-measure of 1 as presented in Table 6. Thus the proposed system performed excellently well when compared to existing systems as presented in the results. 


\begin{tabular}{|c|c|c|c|c|}
\hline \multicolumn{4}{|c|}{ [9. Prediction table } & \begin{tabular}{l|l|l|l|l|} 
& \\
\end{tabular} \\
\hline Index & Actual & Predicted & Error & CM1 \\
\hline 1 & 0.0 & 0.0 & & 0.008483625022 \\
\hline 2 & 0.0 & 0.0 & & $0.008597336281 \ldots$ \\
\hline 3 & 0.0 & 0.0 & & $0.008609725244 \ldots$ \\
\hline 4 & 0.0 & 0.0 & & $0.008481772364 \ldots$ \\
\hline 5 & 0.0 & 0.0 & & $0.008602525524 \ldots$ \\
\hline 6 & 0.0 & 0.0 & & $0.008508532346 \ldots$ \\
\hline 7 & 0.0 & 0.0 & & $0.008719027758 \ldots$ \\
\hline 8 & 0.0 & 0.0 & & $0.008248707470 \ldots$ \\
\hline 9 & 0.0 & 0.0 & & $0.008438132351 \ldots$ \\
\hline 10 & 0.0 & 0.0 & & $0.008625578319 \ldots$ \\
\hline 11 & 0.0 & 0.0 & & $0.008234889176 \ldots$ \\
\hline 12 & 0.0 & 0.0 & & $0.008641878535 \ldots$ \\
\hline 13 & 0.0 & 0.0 & & $0.008185616481 \ldots$ \\
\hline 14 & 0.0 & 0.0 & & $0.008578355388 \ldots$ \\
\hline 15 & 0.0 & 0.0 & & $0.008478415060 \ldots$ \\
\hline 16 & 1.0 & 1.0 & & $0.009761450270 \ldots$ \\
\hline 17 & 1.0 & 1.0 & & $0.009109925152 \ldots$ \\
\hline 18 & 1.0 & 1.0 & & $0.009278918287 \ldots$ \\
\hline 19 & 1.0 & 1.0 & & $0.009060988095 \ldots$ \\
\hline 20 & 1.0 & 1.0 & & $0.009404838275 \ldots$ \\
\hline 21 & 1.0 & 1.0 & & $0.009510573862 \ldots$ \\
\hline 22 & 1.0 & 1.0 & & $0.008988017482 \ldots$ \\
\hline 23 & 1.0 & 1.0 & & $0.009267976482 \ldots$ \\
\hline 24 & 1.0 & 1.0 & & $0.009112681138 \ldots$ \\
\hline 25 & 1.0 & 1.0 & & $0.009648493080 \ldots$ \\
\hline 26 & 1.0 & 1.0 & & $0.009122305271 \ldots$ \\
\hline 27. & 1.0 & 1.0 & & 0.009066031912 \\
\hline 28 & 1.0 & 1.0 & & $0.009227015345 \ldots$ \\
\hline 29 & 1.0 & 1.0 & & $0.009222353270 \ldots$ \\
\hline 30 & 1.0 & 1.0 & & $0.009720964720 \ldots$ \\
\hline
\end{tabular}

Table 6: Prediction Table for Iris Dataset

From Fig.9, which predicts for a single instance, it would also be observed that even though there was a misclassification using the traditional approach yet with the aid of our proposed model, we were able to correctly classify the instance using our confusability measurement threshold to the correct class-Iris-Versicolor. It should be noted that using the traditional approach of classification whereby only the truth membership is used, it will be concluded and labeled or assign to the class Iris-Virginica but with the aid of our confusability measurement it was classified correctly to be Iris-Versicolor. Iris-Versicolor and Iris- Virginica are designated as 0 and 1 respectively.

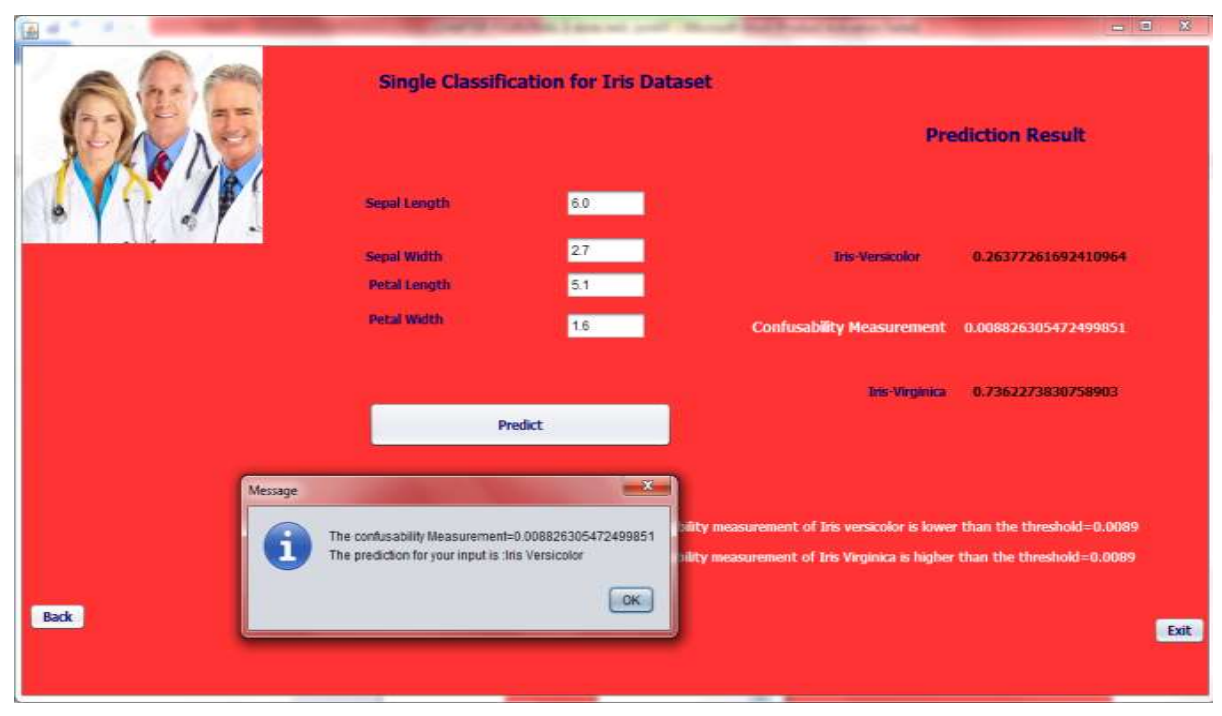

Fig. 9: Single classification interface for Iris 


\section{(b) Discussion of Wine Results}

Our model or approach was applied to the wine dataset. The wine dataset was identified to be slightly overlapped with an overlap ratio of $7.30 \%$ as opined by existing system of (Haitao et al (2013)).

When our proposed system was tested using the wine dataset, the confusability measurement was computed for the three classes of wine and it was observed that the correct class is the class with the lowest confusability measurement. The prediction table for wine dataset used for testing showing the confusability of the three classes for every instance is shown in Table A.1. This observation gives credence to the fact that the higher the confusability measurement the more unlikely the correctness of the predicted class.

\section{(c) Discussion of Diabetes Results}

Diabetes dataset from $\mathrm{UCl}$ has the dual problem of class imbalance and overlapping. Haitao et al (2013) estimated the overlapping ratio of the dataset to be $24.3 \%$. We also applied the dataset to our model. The performance evaluation of our proposed system with appropriate performance metrics is shown in Figure.A.1. The work of Haitao et al (2013) performed resampling on the dataset since the dataset is not only overlapped but also imbalanced.

From the performance analysis of our approach on this dataset, it could be observed that it did not perform very well when compared to existing system using resampling but on the contrary perform better than the existing systems without resampling. This is traceable to the fact that we did not perform any resampling on the data or could be due to other data characteristics.

This result gives credence to the fact that apart from overlap, there are other data characteristics that can impact negatively on the performance of classifiers as opined by Sharma et al (2016). Our approach did not perform very well on a dataset that is not balanced and as such resampling should be done first before applying the proposed model in order to handle such situations. Future work would handle this concept.

\section{(d) Discussion of Indian Liver Patient Dataset Results}

The Indian Liver Patient Dataset (ILPD) has two classes designated as 1 (Positive) and 2 (Negative) which are observed to be overlapping hence could be confusing in terms of prediction. This dataset has a dual problem of class imbalance and overlap problem.

Our proposed model was used for the ILPD dataset; a confusability measurement threshold of 0.003278 was estimated which was clearly used to assign the class labels accordingly. Confusability measurement is greater than 0.003278 for all positive instances while negative instances should be less than 0.003278 and if any of the prediction goes contrary, such prediction should be updated. The class label " 1 " is used to denote liver disease patient while "0" is used to denote non-liver patient.

It could be seen that with our approach the accuracy of the model is increased from $72.45 \%$ to $96.41 \%$ as presented in the results which is about $24 \%$ increase.

\subsubsection{Discussion on External Confusability Results}

This is the kind of overlapping or confusability that exists between two different datasets. The external confusability considered two datasets, Indian Liver Patient Dataset (ILPD) and Hepatitis dataset. In order to implement this and justifiably say that there is confusability in the datasets in terms of diagnosis or classification, the test dataset of Hepatitis was fed into the ILPD classifier (neural network). We observed a specificity of $100 \%$ while the sensitivity was low. This observation is in consonance with medical theory that all Hepatitis is Liver Disorder. But when ILPD testing instances was fed into Hepatitis classifier, a low or near zero specificity was observed. The research experimented with two approaches: 
(a) Using the traditional model of neural network all positive Indian Liver Patient Dataset was labeled as class I designated as '1" while all positive Hepatitis disease as class II designated as '0' which is referred to as "Confusable Diseases Dataset". The testing dataset is made of $30 \%$ of both class I and class II. The performance of the traditional system in diagnosing confusable disease shows a classification accuracy of $93.5897 \%$ and F-measure of 0.936 shown in Figure 4.40 while an ROC Area under Curve of 0.91 as recorded in the result.

(b) Using the proposed system with the confusable disease test data which was also used in the traditional MLP model of the neural networks, the prediction table showing the confusability measurement of the ILPD testing instances on Hepatitis and ILPD classifier is shown in Tables A.2 and A.3 while the confusability measurement of Hepatitis testing instances on ILPD and Hepatitis classifier is shown in Tables A.4 and A.5.

The Confusability measurement on testing confusable disease instance on Hepatitis and ILPD classifier is shown in Tables A.6 and A.7. It was observed for every instance of true Liver Disorder (ILPD), the confusability measurement is low on ILPD but very high for Hepatitis. It is also observed for every instance of true Hepatitis, the confusability measurement is low on Hepatitis but high for Liver Disorder. With the use of the confusability measurement in decision making, one is able to differentiate between Liver Disorder (ILPD) and Hepatitis. The system recorded an F-measure of 0.9607 and an accuracy of $96.15 \%$ which clearly indicates the high generalization ability of our model as it is better in performance than using the traditional approach. It was adequately used to differentiate between Liver Disorder (ILPD) and Hepatitis taking either Liver Disorder (ILPD) or Hepatitis as the index disease.

\section{CONCLUSION}

A new model to handle overlapping problem in data classification is presented using Neutrosophic-based Bayesian Neural Network (NBBNN) approach and its performance shows a high potential in alleviating the problem of overlapping in classification problems. In this research work the proposed system takes features of each of the datasets presented and a confusability measurement is calculated alongside the predicted class such that it is used to make appropriate decisions on instances which would have been difficult to predict due to overlapping regions.

\section{REFERENCES}

1. Adeniji K.A and Anjorin A.S. (2002). Histopathological Assessment of the pattern of liver cirrhosis in a tropical population. Afr J Med Sci 2002; 31: 367-9

2. Ansari A. Q, Biswas R. and Aggarwal S. (2013). Neutrosophic classifier: An extension of fuzzy classifier. Applied Soft Computing, 13(1) 563-573.

3. Ashwani Kumar and Neelam Sahu. (2017). Categorization of Liver Disease Using Classification Techniques, International Journal for Research in Applied Science \& Engineering Technology (IJRASET) 826

4. Dhamodharan S. (2014) . Liver Diseases Prediction Using Bayesian Classification. National Conference on Advanced Computing, Applications \& Technologies, ISSN: 2320-0790, 1-3, 2014.

5. Drăgulescu Doina, and Albu Adriana(2007). Medical Predictions System volume 4(3).

6. Gulia, Vohra R. and Rani P. (2014) . Liver Patient Classification Using Intelligent Techniques, International Journal of Computer Science and Information Technologies, 5 (4) , 5110-5115

7. Jankisharan Pahareeya, Rajan Vohra, Jagdish makhijani and Sanjay Patsariya (2014). Liver Patient Classification Using Intelligence Techniques. International journal of advanced research in computer science and software enginerring.4 (2).

8. Javad Salimi and Sartakht, J. S. (2011). Hepatitis disease diagnosis using a novel hybrid method, Elsevier, 570-579. 
9. Kavitha B., Karthikeyan S. and Sheeba Maybell P (2011): An ensemble design of intrusion detection system for handling uncertainty using Neutrosophic Logic Classifier. 28 (2012) 88-96

10. Khorashadizade Nahid and Rezaei Hassan (2015): A New method for rapid diagnosis of Hepatitis disease based on reduction feature and machine learning-Journal of Advanced Computer Science and Technology . 4(1) 148-155.

11. Mohammed Abdullah Alghambi, Sunnil G. Bhirud and Afshar M. Alam(2015). Physician's decision process for disease diagnosis of overlapping syndrome in liver disease using soft computing. International Journal of Soft Computing and Engineering.(IJSCE).4(6)28-33.

12. Nwokediuko S.C, Osuala P.C, Uduma U.V, Alaneme A.K, Onwuka C.C, and Mesigo C. (2013). Pattern of Liver disease in Nigeria Tertiary Hospital. Niger J Clin Pract. 16,339-42

13. Obeidat Ibrahim, Al-khasawneh Almad, Bsoul Maohammad (2013). Computer based clinical decision support system for hepatitis disease diagnosis-International journal of advancements in computing technology. 5 ; 1522.

14. Pooja Shrivastara and Yukti Kishawari (2016). An Efficient Classifier using Multilayer Perceptron for Classification of Liver patient. International Journal for Research in Applied Science \& Engineering Technology (IJRASET). 4(6); 646-648.

15. Ramana B. V. and Surendra Prasad Babu M. (2012). Liver Classification Using Modified Rotation Forest. International Journal of Engineering Research and Development, 1(6) 17-24, 2012.

16. Tahseen A. Jilani, Huda Yasin and Madiha Mohammad Yasin (2011). PCA-ANN for classification of Hepatitis $C$ patients- International journal of computer applications

17. Vijayarani S. and Dhayanand S. (2015). Liver Disease Prediction using SVM and Naïve Bayes Algorithms. International Journal of Science, Engineering and Technology Research (IJSETR) 4(4).

18. Zadeh L.A. (1996). Fuzzy logic = Computing with Words. IEEE Trans. on Fuzzy Systems, 4, 103-111.

\section{About the Authors}

* Dr. Okpako Abugor Ejaita: A lecturer in the Department of Mathematical Sciences, Edwin Clark University, Kiagbodo, Delta State, Nigeria.

* Dr.(Mrs) Rita Ako: A lecturer in the Department of Mathematical Sciences, Edwin Clark University, Kiagbodo, Delta State,Nigeria. 
Table A.1: Prediction Table for Wine Dataset

\begin{tabular}{|c|c|c|c|c|c|c|c|}
\hline & & & & & & 푸 & \\
\hline Index & Actual & Predicted & Error & CMI 1 & CM2 & $\mathrm{CH} 3$ & \\
\hline 1 & 2.0 & 2.0 & & 0.0097128688881103898 & 0.009501743785751415 & 0.019214612666855202 & 1 \\
\hline 2 & 20 & 2.0 & & 0.01107311355028473 & 0.00663694242103452 & 0.01771005597131936 & \\
\hline 3 & 2.0 & 2.0 & & 0.013161635415166018 & 0.005409232083213755 & 0.018570867498379995 & \\
\hline 4 & 20 & 2.0 & & 0.013131267207673547 & 0.005396278495652718 & 0.018527545703326265 & \\
\hline 5 & 20 & 2.0 & & 0.012610978286357044 & 0.005531091120370979 & 0.018142069406728023 & \\
\hline 6 & 20 & 2.0 & & 0.01266239327008778 & 0.005511597656805423 & 0.018173990926893202 & \\
\hline 7 & 2.0 & 2.0 & & 0.01238764600794362 & 0.005539543478674358 & 0.01792718948661809 & \\
\hline 8 & 2.0 & 2.0 & & 0.011477632483233857 & 0.00634179234168164 & 0.017819424824915386 & \\
\hline 9 & 20 & 2.0 & & 0.015736968269135287 & 0.0048763533865730598 & 0.020613322134865886 & \\
\hline 10 & 2.0 & 2.0 & & 0.008871011987163557 & 0.009525857479542044 & 0.0183968694667056 & \\
\hline 11 & 2.0 & 2.0 & & 0.013774978716755037 & 0.005306835015386091 & 0.01908181373214113 & \\
\hline 12 & 2.0 & 2.0 & & 0.015606672308546399 & 0.004903526915372103 & 0.0205101992239185 & \\
\hline 13 & 2.0 & 20 & & 0.012083548514522757 & 0.005968564218170824 & 0.018052112732693693 & \\
\hline 14 & 2.0 & 2.0 & & 0.05751926375322258 & 0.00238067709925327 & 0.059899940852475964 & \\
\hline 15 & 2.0 & 2.0 & & 0.017195167983031445 & 0.004645515950426726 & 0.02184068393345817 & \\
\hline 16 & 2.0 & 2.0 & & 0.010918690791965835 & 0.006800270131064545 & 0.01771896092303049 & \\
\hline 17 & 2.0 & 2.0 & & 0.016166110778671805 & 0.005952945751107941 & 0.022119056529779857 & \\
\hline 18 & 1.0 & 1.0 & & 0.004625991085651315 & 0.013355481066659736 & 0.008729489981008531 & \\
\hline 19 & 1.0 & 1.0 & & 0.0037698085251144375 & 0.011640764998717024 & 0.007870956473602475 & \\
\hline 20 & 1.0 & 1.0 & & 0.029467243280428934 & 0.03397996520891444 & 0.004512721928485619 & \\
\hline 21 & 1.0 & 1.0 & & 0.004111490720754896 & 0.011830288100688424 & 0.0077187973799335285 & \\
\hline 22 & 1.0 & 1.0 & & 0.0038479744194338528 & 0.011787617864236322 & 0.007939643444802358 & \\
\hline 23 & 1.0 & 1.0 & & 0.009883936483035871 & 0.017221015239183624 & 0.007337078756147752 & \\
\hline 24 & 1.0 & 1.0 & & 0.003663278863539232 & 0.01165395403128533 & 0.007990675167746208 & \\
\hline 25 & 1.0 & 1.0 & & 0.004235768833035447 & 0.011985410925031914 & 0.007749642091996467 & \\
\hline 26 & 1.0 & 1.0 & & 0.003624061648274446 & 0.011560907744265658 & 0.007936846095991101 & \\
\hline 27 & 1.0 & 1.0 & & 0.0037013818353253125 & 0.011641215272107264 & 0.007939833436781951 & \\
\hline 28 & 1.0 & 1.0 & & 0.0037286938916323287 & 0.011601274806126494 & 0.007872580914494165 & \\
\hline 29 & 1.0 & 1.0 & & 0.00260198490922535553 & 0.10221615681736651 & 0.09961417190813115 & \\
\hline 30 & 1.0 & 1.0 & & 0.004107226777803508 & 0.011777180719389158 & 0.007669953941505428 & \\
\hline 31 & 1.0 & 1.0 & & 0.011023059187923012 & 0.017586571143967333 & 0.006563511956044321 & \\
\hline 32 & 1.0 & 1.0 & & 0.4056979003703842 & 0.4065924607161602 & 8.945603457759521E-4 & \\
\hline 33 & 1.0 & 1.0 & & 0.0054032794945171325 & 0.012986827302645665 & 0.007583547808128532 & \\
\hline 34 & 1.0 & 1.0 & & 0.020996283968484852 & 0.027140378653826347 & 0.006144094685341384 & \\
\hline 35 & 1.0 & 1.0 & & 0.01880761257109753 & 0.023823615701305756 & 0.005016003130208335 & \\
\hline 36 & 1.0 & 1.0 & & 0.0042597262000002922 & 0.011766366587489752 & 0.00750664038748694 & \\
\hline 37 & 1.0 & 1.0 & & 0.00564835375181294 & 0.01325736309456127 & 0.007609009342748441 & \\
\hline 38 & 1.0 & 1.0 & & 0.003785572443715024 & 0.011768551568764662 & 0.007982979125049638 & \\
\hline 39 & 1.0 & 1.0 & & 0.0037652736738995607 & 0.011631308709969757 & 0.007866035036070196 & \\
\hline 40 & 0.0 & 0.0 & & 0.03091612930674381 & 0.026631289471931052 & 0.004284839834812648 & \\
\hline 41 & 0.0 & 0.0 & & 0.020489700040213443 & 0.015937076113749504 & 0.004552623926463828 & \\
\hline 42 & 0.0 & 0.0 & & 0.048376720080390734 & 0.04041644475337913 & 0.007960275327011712 & \\
\hline 43 & 0.0 & 1.0 & + & 0.9333361183959393 & 0.9364933834299378 & 0.00315726503399838 & \\
\hline 44 & 0.0 & 0.0 & & 0.015267373130403605 & 0.010752029639135685 & 0.00451534349126792 & \\
\hline
\end{tabular}


Table A.2: ILPD Prediction

\begin{tabular}{|c|c|c|c|c|c|c|c|c|c|c|c|}
\hline \multirow{2}{*}{\multicolumn{6}{|c|}{ Table A.2: ILPD Prediction $\quad \square|\square|-\gamma \mid$}} & & \\
\hline & & & & & & \multirow{3}{*}{\multicolumn{2}{|c|}{ 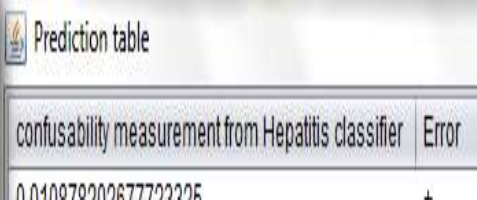 }} & \multirow{3}{*}{$\begin{array}{l}\text { Actual } \\
1.0\end{array}$} & \multirow{2}{*}{\multicolumn{2}{|c|}{ Predicted Inde }} & \\
\hline Index & Actual & Predicer & & confivsability measurementusing Liver disorder classfifier & & & & & & & \\
\hline 1 & 1.0 & 1.0 & & 0.0032767505577099827 & 1) & & & & 0.0 & 1 & i \\
\hline 2 & 1.0 & 1.0 & & 0.0035302099427305507 & & 00108014506330229402 & + & 1.0 & 0.0 & 2 & \\
\hline 3 & 1.0 & 1.0 & & 0.0077616255749385018 & & 0.014559828199727343 & + & 1.0 & 0.0 & 3 & \\
\hline 4 & 1.0 & 1.0 & + & 0.0035212193930100979 & & 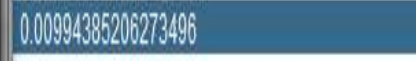 & + & 10 & 00 & 4 & \\
\hline 5 & 1.0 & 0.0 & & 0.003467526773420057 & & D.0094872089915159551 & + & 10 & 0.0 & 5 & \\
\hline 6 & 1.0 & 1.0 & & D.00399970564608553934 & & 0.011682499970442461 & + & 10 & 0.0 & 6 & \\
\hline 1 & 1.0 & 1.0 & & 0.056551580891105501 & & 0.057785959790985415 & + & 10 & 0.0 & 7 & \\
\hline 8 & 1.0 & 1.0 & & 0.004120640813635722 & & 0.0136833030354595720 & + & 10 & 0.0 & 8 & \\
\hline 9 & 1.0 & 1.0 & & 0.0038284403771241919394 & & 0.013978799611361474 & + & 1.0 & 0.0 & 9 & \\
\hline 10 & 1.0 & 1.0 & & D.003900411228999479 & & 0.00638444461125312828 & & 1.0 & 1.0 & 10 & \\
\hline 11 & 1.0 & 0.0 & + & 0.003263974688480108775 & & 0.000554234643565303055 & + & 1.0 & 0.0 & 11 & \\
\hline 12 & 1.0 & 1.0 & & 0.005203537201785501 & & 0.0186021170578905 & + & 1.0 & 0.0 & 12 & \\
\hline 13 & 1.0 & 1.0 & & 0.00456530790977191 & & 0016047468620032502 & + & 1.0 & 0.0 & 13 & \\
\hline 14 & 1.0 & 1.0 & & 0.003555553330764711025 & & 0.012356352606517814 & + & 10 & 0.0 & 14 & \\
\hline 15 & 1.0 & 1.0 & & 0.0049191664459447717 & & 0.044625651560728802 & + & 10 & 0.0 & 15 & \\
\hline 16 & 1.0 & 1.0 & & 0.053415746645601114 & & 0.067988777433997069 & + & 1.0 & 0.0 & 16 & \\
\hline 17 & 1.0 & 1.0 & & 0.0502209888111986688 & & 0.015623380407178988008 & + & 1.0 & 0.0 & 17 & \\
\hline 18 & 0.0 & 1.0 & & 0.003555001245890017 & & 0.011233982644182272 & & 0.0 & 0.0 & 18 & \\
\hline 19 & 1.0 & 1.0 & & 0.010353120465284471 & & 0.039394811359472265 & + & 1.0 & 0.0 & 19 & \\
\hline 20 & 1.0 & 1.0 & & 0.010490017047508133 & & 0.0271426377355517883 & + & 1.0 & 0.0 & 20 & \\
\hline 21 & 1.0 & 1.0 & & 0.004564341216098833 & & 0.08106404477555225 & + & 1.0 & 0.0 & 21 & \\
\hline 22 & 1.0 & 1.0 & & 0.00373991560600995523 & & O.009909075588083359 & + & 10 & 0.0 & 22 & \\
\hline 23 & 1.0 & 1.0 & & 0.003343418029204489996 & & 09100093535541450161 & + & 1.0 & 0.0 & 23 & \\
\hline 24 & 1.0 & 1.0 & & 0.006208743087557478 & & 0.010139910503880255758 & + & 1.0 & 0.0 & 24 & \\
\hline 25 & 1.0 & 1.0 & & 0.01354305304311787 & & 0.0304700252312986005 & + & 1.0 & 0.0 & 25 & \\
\hline 26 & 1.0 & 1.0 & & 0.031600446646528756 & & 0.033944600301883134 & + & 1.0 & 0.0 & 26 & \\
\hline 27 & 1.0 & 1.0 & & 0.0107383737868653939 & & 0.02223014741219942 & + & 1.0 & 0.0 & 27 & \\
\hline 28 & 1.0 & 1.0 & & 0.0074565645599633138 & & 0.016481486371652143 & + & 10 & 0.0 & 28 & \\
\hline 29 & 1.0 & 1.0 & & 0.003588087639648453505 & & 0.0108998885529794619 & + & 10 & 0.0 & 29 & \\
\hline 30 & 1.0 & 0.0 & & 0.00337732556428567716 & & 0.0098865202554031857 & + & 1.0 & 0.0 & 30 & \\
\hline 31 & 1.0 & 1.0 & & 0.005539147006596795 & & 0.0178843802629405022 & + & 1.0 & 0.0 & 31 & \\
\hline 32 & 1.0 & 1.0 & & 0.00332999988320878552 & & 0.012247929440418022 & + & 1.0 & 0.0 & 32 & \\
\hline 33 & 1.0 & 1.0 & & 0.0040832442651103085 & , & 0.012466026478821733 & + & 1.0 & 0.0 & 33 & , \\
\hline
\end{tabular}




\section{Prediction Table for ILPD test Instances on ILPD classifier and Hepatitis classifier showing the difference in values}

Table A.4: Hepatitis Prediction

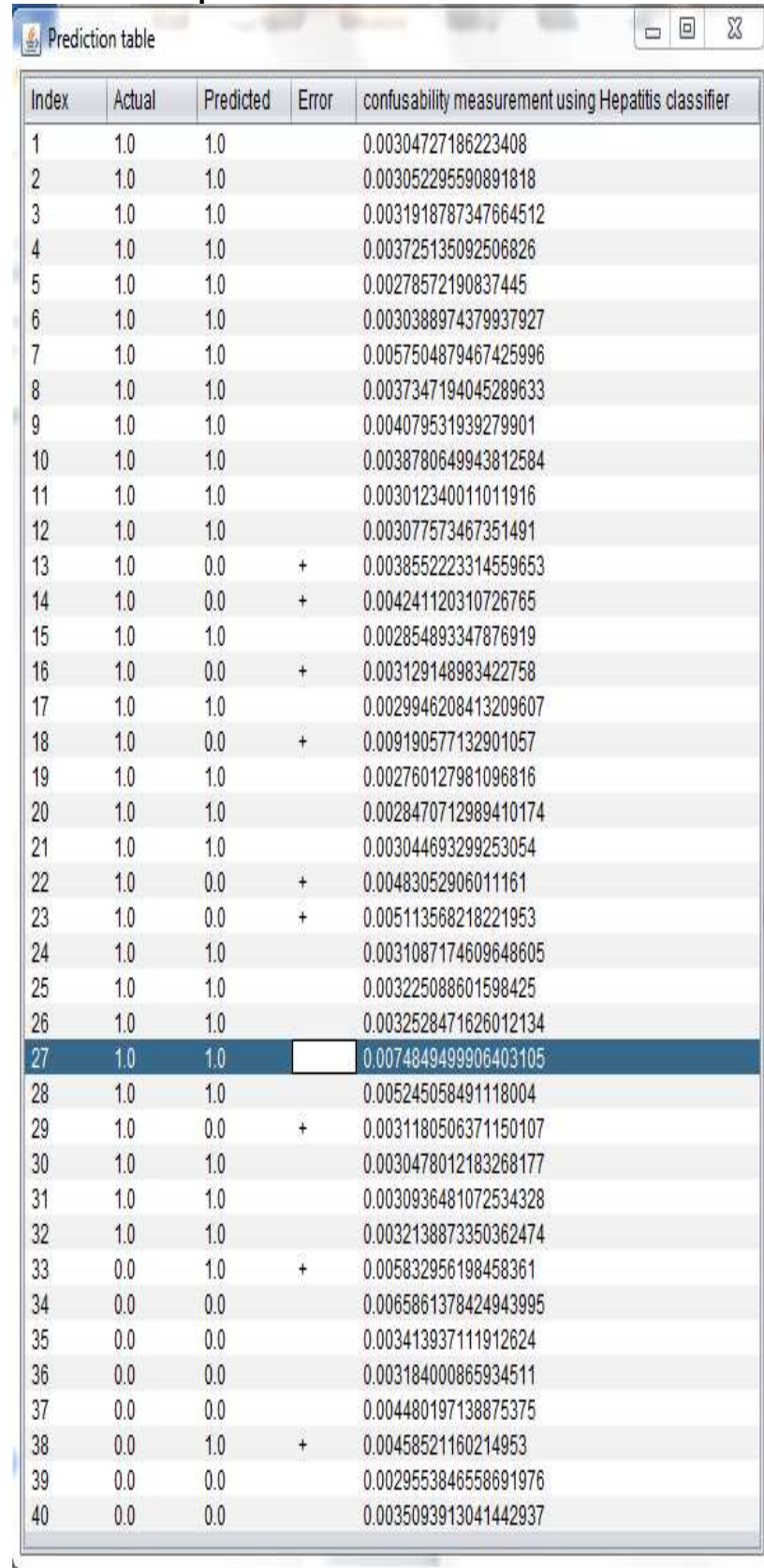

Table A.5: ILDP Prediction

\begin{tabular}{|c|c|c|c|c|}
\hline \multicolumn{2}{|l|}{ (4) Prediction table } & & & 口 回 88 \\
\hline confusability measurement & Actual & Predicted & Error & Index \\
\hline 0.005888169780195351 & 1.0 & 1.0 & & 1 \\
\hline 0.005665098565105997 & 1.0 & 1.0 & & 2 \\
\hline 0.175007603821703 & 1.0 & 1.0 & & 3 \\
\hline 0.17986922149482065 & 1.0 & 1.0 & & 4 \\
\hline 0.1764044329855341 & 1.0 & 1.0 & & 5 \\
\hline 0.005868940129063249 & 1.0 & 1.0 & & 6 \\
\hline 0.014696809452026338 & 1.0 & 1.0 & & 7 \\
\hline 0.15858682646740552 & 1.0 & 1.0 & & 8 \\
\hline 0.17620412918569262 & 1.0 & 1.0 & & 9 \\
\hline 0.006048535140585232 & 1.0 & 1.0 & & 10 \\
\hline 0.17985289319451592 & 1.0 & 1.0 & & 11 \\
\hline 0.1792579635356606 & 1.0 & 1.0 & & 12 \\
\hline 0.1048351863910566 & 1.0 & 1.0 & & 13 \\
\hline 0.13474067723630134 & 1.0 & 1.0 & & 14 \\
\hline 0.17277306707828655 & 1.0 & 1.0 & & 15 \\
\hline 0.007924152658807526 & 1.0 & 1.0 & & 16 \\
\hline 0.0054534328129404175 & 1.0 & 1.0 & & 17 \\
\hline 0.17616505899087476 & 1.0 & 1.0 & & 18 \\
\hline 0.16135482302313875 & 1.0 & 1.0 & & 19 \\
\hline 0.006366572402044968 & 1.0 & 1.0 & & 20 \\
\hline 0.005769240328860503 & 1.0 & 1.0 & & 21 \\
\hline 0.006585306141969949 & 1.0 & 1.0 & & 22 \\
\hline 0.008017600250187051 & 1.0 & 1.0 & & 23 \\
\hline 0.16958787611417758 & 1.0 & 1.0 & & 24 \\
\hline 0.006561294091649739 & 1.0 & 1.0 & & 25 \\
\hline 0.16450509857372508 & 1.0 & 1.0 & & 26 \\
\hline 0.0064444424586684696 & 1.0 & 1.0 & & 27 \\
\hline 0.17685054933422029 & 1.0 & 1.0 & & 28 \\
\hline 0.1657077427266238 & 1.0 & 1.0 & & 29 \\
\hline 0.005624208622253879 & 1.0 & 1.0 & & 30 \\
\hline 0.007668537460987723 & 1.0 & 1.0 & & 31 \\
\hline 0.17987927576378948 & 1.0 & 1.0 & & 32 \\
\hline 0.13716709773514724 & 0.0 & 1.0 & + & 33 \\
\hline 0.009442045325437465 & 0.0 & 1.0 & + & 34 \\
\hline 0.00835564939852873 & 0.0 & 1.0 & + & 35 \\
\hline 0.008902765528545853 & 0.0 & 1.0 & + & 36 \\
\hline 0.11351105675995199 & 0.0 & 1.0 & + & 37 \\
\hline 0.17331210557395882 & 0.0 & 1.0 & + & 38 \\
\hline 0.17989090349967984 & 0.0 & 1.0 & + & 39 \\
\hline 0.007752203679173397 & 0.0 & 1.0 & + & 40 \\
\hline
\end{tabular}




\section{Prediction Table for Hepatitis test Instances on Hepatitis classifier and ILPD classifier showing the difference in values}

Table A.6:

\begin{tabular}{|c|c|c|c|c|}
\hline \multicolumn{2}{|c|}{ Prediction table } & \multirow[b]{2}{*}{ Predicted } & & 미 回 \\
\hline Index & Actual & & Error & confusability me... \\
\hline 20 & 10 & 0 & + & 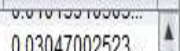 \\
\hline 30 & 1.0 & 0.0 & + & 003394468030 \\
\hline 31 & 1.0 & 0.0 & + & $0.02223014741 \ldots$ \\
\hline 32 & 1.0 & 0.0 & + & $0.01648148637 \ldots$ \\
\hline 33 & 1.0 & 0.0 & + & $0.01089488352 \ldots$ \\
\hline 34 & 1.0 & 0.0 & + & $0.00968152905 \ldots$ \\
\hline 35 & 1.0 & 0.0 & + & $0.01788438262 \ldots$ \\
\hline 36 & 1.0 & 0.0 & + & $0.01224972944 \ldots$ \\
\hline 37 & 1.0 & 0.0 & + & $0.01246626478 \ldots$ \\
\hline 38 & 1.0 & 0.0 & + & $0.00963328015 \ldots$ \\
\hline 39 & 1.0 & 0.0 & + & $0.01237009717 \ldots$ \\
\hline 40 & 1.0 & 0.0 & + & $0.02147810603 \ldots$ \\
\hline 41 & 1.0 & 0.0 & + & $0.07570781422 \ldots$ \\
\hline 42 & 1.0 & 0.0 & + & $0.05539180789 \ldots$ \\
\hline 43 & 1.0 & 0.0 & + & $0.04303149536 \ldots$ \\
\hline 44 & 1.0 & 0.0 & + & $0.04912136877 \ldots$ \\
\hline 45 & 1.0 & 0.0 & + & $0.01347249338 \ldots$ \\
\hline 46 & 1.0 & 0.0 & + & $0.03352491915 \ldots$ \\
\hline 47 & 0.0 & 1.0 & + & $0.00304727186 \ldots$ \\
\hline 48 & 0.0 & 1.0 & + & $0.00305229559 \ldots$ \\
\hline 49 & 0.0 & 1.0 & + & $0.00319187873 \ldots$ \\
\hline 50 & 0.0 & 1.0 & + & $0.00372513509 . \ldots$ \\
\hline 51 & 0.0 & 1.0 & + & $0.00278572190 \ldots$ \\
\hline 52 & 0.0 & 1.0 & + & $0.00303889743 \ldots$ \\
\hline 53 & 0.0 & 1.0 & + & $0.00575048794 \ldots$ \\
\hline 54 & 0.0 & 1.0 & + & $0.00373471940 \ldots$ \\
\hline 55 & 0.0 & 1.0 & + & $0.00407953193 \ldots$ \\
\hline 56 & 0.0 & 1.0 & + & $0.00387806499 \ldots$ \\
\hline 57 & 0.0 & 1.0 & + & $0.00301234001 \ldots$ \\
\hline 58 & 0.0 & 1.0 & + & $0.00307757346 \ldots$ \\
\hline 59 & 0.0 & 0.0 & & $0.00385522233 \ldots$ \\
\hline 60 & 0.0 & 0.0 & & $0.00424112031 \ldots$ \\
\hline 61 & 0.0 & 1.0 & + & $0.00285489334 \ldots$ \\
\hline 62 & 0.0 & 0.0 & & $0.00312914898 \ldots$ \\
\hline 63 & 0.0 & 1.0 & + & $0.00299462084 \ldots$ \\
\hline 64 & 0.0 & 0.0 & & $0.00919057713 \ldots$ \\
\hline 65 & 0.0 & 1.0 & + & $0.00276012798 \ldots$ \\
\hline 66 & 0.0 & 1.0 & + & $0.00284707129 \ldots$ \\
\hline 67 & 0.0 & 1.0 & + & $0.00304469329 \ldots$ \\
\hline 68 & 0.0 & 0.0 & & $0.00483052906 \ldots$ \\
\hline 69 & no & no & & n0055113568821 T \\
\hline
\end{tabular}

Table A.7:

\begin{tabular}{|c|c|c|c|c|}
\hline \multicolumn{4}{|c|}{ (1) Prediction table using NBDSS(Our Model) } & \multirow{3}{*}{ 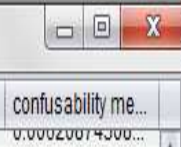 } \\
\hline Index & Actual & Predicted & Error & \\
\hline 20 & 1.0 & 1.0 & & \\
\hline 29 & 1.0 & 1.0 & & $0.01354305304 \ldots$ \\
\hline 30 & 1.0 & 1.0 & & $0.03166041464 \ldots$ \\
\hline 31 & 1.0 & 1.0 & & $0.01073837378 \ldots$ \\
\hline 32 & 1.0 & 1.0 & & $0.00745656459 \ldots$ \\
\hline 33 & 1.0 & 1.0 & & $0.00358887639 \ldots$ \\
\hline 34 & 1.0 & 0.0 & & $0.00334732554 \ldots$ \\
\hline 35 & 1.0 & 1.0 & & $0.00553914760 \ldots$ \\
\hline 36 & 1.0 & 1.0 & & $0.00342919983 \ldots$ \\
\hline 37 & 1.0 & 1.0 & & $0.00408324426 \ldots$ \\
\hline 38 & 1.0 & 0.0 & & $0.00330202388 \ldots$ \\
\hline 39 & 1.0 & 1.0 & & $0.00421156660 \ldots$ \\
\hline 40 & 1.0 & 1.0 & & $0.02011947619 \ldots$ \\
\hline 41 & 1.0 & 1.0 & & $0.04797905933 \ldots$ \\
\hline 42 & 1.0 & 1.0 & & $0.05102679762 \ldots$ \\
\hline 43 & 1.0 & 1.0 & & $0.02864203757 \ldots$ \\
\hline 44 & 1.0 & 1.0 & & $0.02781655886 \ldots$ \\
\hline 45 & 1.0 & 1.0 & & $0.00353047890 \ldots$ \\
\hline 46 & 1.0 & 1.0 & & $0.01549162007 \ldots$ \\
\hline 47 & 0.0 & 1.0 & & $0.00588816978 \ldots$ \\
\hline 48 & 0.0 & 1.0 & & $0.00566509856 \ldots$ \\
\hline 49 & 0.0 & 1.0 & & $0.17500760382 \ldots$ \\
\hline 50 & 0.0 & 1.0 & & $0.17986922149 \ldots$ \\
\hline 51 & 0.0 & 1.0 & & $0.17640443298 \ldots$ \\
\hline 52 & 0.0 & 1.0 & & $0.00586894012 \ldots$ \\
\hline 53 & 0.0 & 1.0 & & $0.01469680945 \ldots$ \\
\hline 54 & 0.0 & 1.0 & & $0.15858682646 \ldots$ \\
\hline 55 & 0.0 & 1.0 & & $0.17620412918 \ldots$ \\
\hline 56 & 0.0 & 1.0 & & $0.00604853514 \ldots$ \\
\hline 57 & 0.0 & 1.0 & & $0.17985289319 \ldots$ \\
\hline 58 & 0.0 & 1.0 & & $0.17925796353 \ldots$ \\
\hline 59 & 0.0 & 1.0 & & $0.10483518639 \ldots$ \\
\hline 60 & 0.0 & 1.0 & & $0.13474067723 \ldots$ \\
\hline 61 & 0.0 & 1.0 & & $0.17277306707 \ldots$ \\
\hline 62 & 0.0 & 1.0 & & $0.00792415265 \ldots$ \\
\hline 63 & 0.0 & 1.0 & & $0.00545343281 \ldots$ \\
\hline 64 & 0.0 & 1.0 & & $0.176165058999 \ldots$ \\
\hline 65 & 0.0 & 1.0 & & $0.16135482302 \ldots$ \\
\hline 66 & 0.0 & 1.0 & & $0.00636657240 \ldots$ \\
\hline 67 & 0.0 & 1.0 & & $0.00576924032 \ldots$ \\
\hline 68 & 0.0 & 1.0 & & $0.00658530614 \ldots$ \\
\hline ha & $n n$ & 10 & & กחก8ח1760025 I \\
\hline
\end{tabular}

Prediction Table for confusable disease test Instances on Hepatitis classifier and ILPD classifier showing the difference in values 